\title{
Revisão Bibliográfica de Pesquisas Brasileiras sobre Equivalência de Estímulos ${ }^{1}$
}

\author{
Juliana Barboza Caetano de Paula ${ }^{2}$ \\ Verônica Bender Haydu \\ Universidade Estadual de Londrina
}

\begin{abstract}
RESUMO - O presente estudo está inserido nas linhas de pesquisa que investigam a equivalência de estímulos. O objetivo principal foi a caracterização das pesquisas sobre equivalência de estímulos produzidas no Brasil e publicadas entre 1997 e 2007. As fontes de materiais bibliográficos consultadas foram resumos de: artigos de periódicos; apresentações em dois eventos da área da Psicologia; e dissertações e teses. Seiscentos e cinquenta e cinco resumos sobre o tema foram localizados: a maioria (516) dos estudos foi realizada com humanos e 253 focalizaram questões educacionais. Muitas tecnologias têm sido produzidas, mas uma maior divulgação é necessária, especialmente na área educacional.
\end{abstract}

Palavras-chave: relações de equivalência; revisão da bibliografia; pesquisas brasileiras.

\section{Literature Review of Brazilian Research on Stimuli Equivalence}

\begin{abstract}
The present study is part of the lines of research that investigate stimuli equivalence. The major aim was to characterize the research on stimuli equivalence produced in Brazil, and published between 1997 and 2007. The sources of bibliographical material consisted of abstracts from: journal articles; presentations in two conventions of the Psychology area; dissertations and theses. Six hundred and fifty five abstracts about the theme were found. The majority of these studies (516) was conducted with humans, and 253 focused on educational issues. Many technologies have been produced, but a greater awareness is needed, especially in education.
\end{abstract}

Keywords: equivalence relations; bibliographic review; Brazilian studies.

Para que se possa compreender os diversos processos comportamentais que ocorrem na interação dos organismos com seu ambiente é necessária a identificação das variáveis que os afetam. Dentre essas variáveis estão as consequências dos comportamentos, as quais determinam a probabilidade de os comportamentos serem repetidos, e os estímulos que estão presentes quando uma dada resposta operante é consequenciada (Skinner, 1953). Na presença do estímulo em que houve o reforço da resposta, ela tenderá a ser repetida e na presença dos estímulos em que a resposta não foi reforçada, ela tenderá a não ser emitida novamente (contingência de três termos; cf. Catania, 1998/1999; Sidman, 2000). O controle de estímulos está vinculado à análise do comportamento conceitual, da emergência de comportamentos novos e do comportamento simbólico (Bortoloti \& de Rose, 2007; Matos, 1999; Wilkinson \& McIlvane, 2001). Dada a importância do conceito de controle de estímulos para a ciência do comportamento, uma grande quantidade de estudos empíricos foi desenvolvida, tendo-se destacado os diferentes aspectos relacionados à formação de classes de estímulos.

1 Este estudo foi possível graças ao apoio do Conselho Nacional de Desenvolvimento Científico e Tecnológico (CNPq). Trabalhos anteriores a este e que lhe deram origem foram apresentados nos seguintes eventos: XV Encontro Anual de Iniciação Científica, XXXVI Reunião Anual de Psicologia e III Encontro Paranaense de Psicologia Escolar e Educacional.

2 Endereço para correspondência: Departamento de Psicologia Geral e Análise do Comportamento, Universidade Estadual de Londrina. Rodovia Celso Garcia Cid, PR 445, KM 380. Caixa Postal 6001. Londrina, PR. CEP 86051-990. Fone/Fax: (43) 3371-4227.E-mail: haydu@uel. br ou julianabarbozadepaula@gmail.com.
As classes de estímulos podem ser formadas por estímulos com propriedades físicas comuns, por estímulos com funções comuns e por estímulos arbitrários (Zentall \& Smeets, 1996). As classes de estímulos arbitrários são consideradas classes de equivalência quando as propriedades definidoras (reflexividade, simetria, transitividade e transitividade simétrica), propostas por Sidman e Tailby (1982), são demonstradas, conforme será descrito a seguir.

Equivalência de estímulos consiste na formação de relações (entre estímulos ou entre estímulos e respostas) que foram explicitamente ensinadas e de outras que, sem ensino direto, emergem depois do aprendizado de pelo menos duas relações condicionais com um membro em comum (Sidman, 1986, 2000). Relações condicionais são estabelecidas quando uma resposta discriminada é colocada sob o controle de estímulos (contingência de quatro termos; cf. Catania, 1998/1999; Sidman, 2000) e um dos estímulos dessa relação participa de uma outra relação condicional, tendo a função de nódulo.

Assim, para desenvolver classes de equivalência, de forma geral, emprega-se o procedimento de pareamento com o modelo, ensinando-se pelo menos duas relações condicionais entre três estímulos (e.g., A1, B1, C1), com, no mínimo, um estímulo em comum e com pelo menos duas possibilidades de escolha a cada tentativa. Por exemplo: (a) diante do estímulo-modelo A1, escolher B1, e não B2, é reforçado (A1:B1B2 - a primeira especificação alfanumérica refere-se ao estímulo-modelo e as outras duas aos estímulos de comparação); (b) diante de A2, escolher B2, e não B1, é reforçado (A2:B1B2); (c) diante de B1, escolher $\mathrm{C} 1$, e 
não $\mathrm{C} 2$, é reforçado (B1:C1C2); (d) diante de B2, escolher $\mathrm{C} 2$, e não $\mathrm{C} 1$, é reforçado (B2:C1C2). As duas possibilidades de escolha e o responder diferencial de acordo com o estímulo-modelo, pode levar à formação, nesse exemplo, das relações condicionais A1B1, B1C1, A2B2 e B2C2. Dado o estabelecimento dessas relações condicionais pelo reforço direto, pode-se testar a emergência de relações que não foram diretamente ensinadas. Se, na ausência de consequências diferenciais explícitas, forem demonstradas as propriedades de reflexividade, de simetria, de transitividade e de simetria da transitividade, pode-se afirmar que se formaram classes de equivalência (Sidman, 1986, 2000; Sidman \& Tailby, 1982).

A formação de relações de equivalência possibilita a expansão das classes, por meio do emparelhamento de um novo estímulo a somente um dos membros, sem a necessidade de emparelhar esse estímulo com todos os elementos da classe (Sidman, Kirk \& Willson-Morris, 1985). A emergência de relações condicionais e o processo de expansão de classes representam uma grande economia para o ensino dos mais diversos comportamentos. Estudos experimentais demonstraram que, com a aplicação desse modelo, pode-se ensinar com alto grau de produtividade comportamentos como os de leitura de palavras e frases, de leitura musical, de escrita, de matemática, de ciências, de habilidades numéricas, de língua de sinais etc. (e.g., Assis \& Galvão, 1996; Bagaiolo \& Micheletto, 2004; Batitucci, 2007; Carmo, Silva \& Figueiredo, 1999; D’Oliveira \& Matos, 1993; de Rose, de Souza \& Hanna, 1996; Figueiredo, Silva, Soares \& Barros, 2001; Medeiros \& Nogueira, 2005; Rossit \& Ferreira, 2003; Souza, Goyos, Silvares \& Saunders, 2007; Stromer, Mackay \& Stoddart, 1992).

Os temas relacionados à aplicação do modelo da equivalência de estímulos, bem como as diversas questões teóricoconceituais que o mesmo suscitou, levaram ao acúmulo de uma grande quantidade de publicações nos mais diversos formatos (e.g., periódicos, livros, capítulos de livros, dissertações e teses, anais de congresso e textos digitalizados disponíveis na WEB). Por exemplo, uma busca na base de dados PsycINFO, realizada em setembro de 2008, com as palavras-chave ${ }^{3}$ : equivalen* relation* OR stimul* equivalen* OR equivalen* stimul*, gerou 612 resumos. Desses resumos, 55 são referentes a livros ou capítulos de livros; 34 , dissertações e teses; e 3 são de coleções eletrônicas. Os 520 resumos restantes correspondem a artigos de periódicos: 79 resumos são de periódicos com apenas um artigo sobre o tema; 138, de periódicos com dois a cinco artigos e 66, de periódicos com seis a nove artigos sobre o tema. Além desses, existem periódicos com pelo menos 10 publicações que atenderam aos critérios selecionados, os quais são apresentados na Figura 1. Essa figura relaciona o nome do periódico com o

3 O símbolo usado nas palavras-chave (*) inclui todas as palavras com o início igual ao que vem antes dele, mas com o restante da palavra variável. Duas palavras próximas significam uma expressão, nesse caso, os trabalhos com as duas palavras escritas em locais diferentes do texto não são contemplados, mas são selecionados apenas os que apresentam as palavras lado a lado. O operador OR indica que a busca deve conter uma expressão ou a outra, ou seja, não é necessária a presença das duas em um mesmo texto. Assim, com essas palavras-chave, foram incluídas as seguintes expressões: equivalence relation(s), equivalent relation(s), stimulus(i) equivalence, equivalent stimulus(i), entre outras. número de resumos encontrados na busca. Foi nela incluído o conjunto de resumos publicados em periódicos brasileiros cuja coluna está destacada.

A partir dos resultados da busca no PsycINFO, observa-se que a maioria dos artigos sobre equivalência de estímulos está distribuída em uma ampla variedade de periódicos publicados nos mais diversos países, como Estados Unidos, Japão, Inglaterra, Canadá, Itália, Espanha, México, entre outros. Além disso, verifica-se, na Figura 1, que os periódicos Psychological Record e Journal of the Experimental Analysis of Behavior, que são dois grandes representantes da publicação internacional da Análise do Comportamento, contam com os maiores números de publicações sobre equivalência de estímulos no período pesquisado. No que se refere à publicação brasileira, supõe-se que apenas uma pequena parte do que é publicado está disponibilizada nessa base, já que foram encontrados trabalhos sobre equivalência de estímulos em apenas cinco periódicos nacionais citados no PsycInfo, quais sejam: Arquivos Brasileiros de Psicologia, Brazilian Journal of Medical and Biological Research, Estudos de Psicologia, Psicologia: Reflexão e Crítica, e Psicologia: Teoria e Pesquisa.

Contrastando com essa aparente pequena produção brasileira sobre equivalência de estímulos, uma busca no diretório de pesquisadores do CNPq, referente ao ano de 2006, revelou sete grupos de pesquisa com a expressão equivalência de estímulos no nome do grupo, no nome da linha de pesquisa ou nas palavras-chave da linha de pesquisa. Esses grupos são coordenados pelos seguintes pesquisadores: Antônio Celso de Noronha Goyos (UFSCAR); Deisy das Graças de Souza (UFSCAR); Gerson Aparecido Yukio Tomanari (USP); José Gonçalves Medeiros (UFSC); Maria Martha Costa Hübner (USP); Olavo de Faria Galvão e Romariz da Silva Barros (UFPA); Verônica Bender Haydu (UEL). Todos esses grupos de pesquisa incluem, além dos pesquisadores, acadêmicos de cursos de pós-graduação e alunos de graduação em programas de iniciação científica e apresentam uma grande produção sobre o tema. Essa produção representa ser um investimento considerável de recursos humanos e de possibilidades para discussões, articulações e o fortalecimento de redes de pesquisadores que podem apresentar contribuições importantes para a Psicologia, maximizando esforços de pesquisa entre seus membros e, principalmente, produzindo conhecimento cientificamente relevante.

Dado o potencial de recursos humanos e a produtividade dos grupos de pesquisa que investem no tema equivalência de estímulos, pode-se constatar que uma pequena parcela do que foi produzido no Brasil sobre esse tema, até a data da última busca, estava disponibilizada pela base de dados PsycINFO, a qual é uma importante fonte de pesquisa na área da Psicologia. Considerando a importância e a extensão dessa produção bibliográfica e a possibilidade de que parte das publicações esteja distribuída e seja desconhecida pelos pesquisadores do restante do país, faz-se necessária a sua organização. Em vista disso, o objetivo do presente estudo foi realizar uma revisão das pesquisas sobre equivalência de estímulos, apresentadas em veículos de divulgação científica brasileiros no período de 1997 a 2007.

Segundo Ferreira (2002), trabalhos de revisão de bibliografia se justificam pela necessidade de divulgação do 


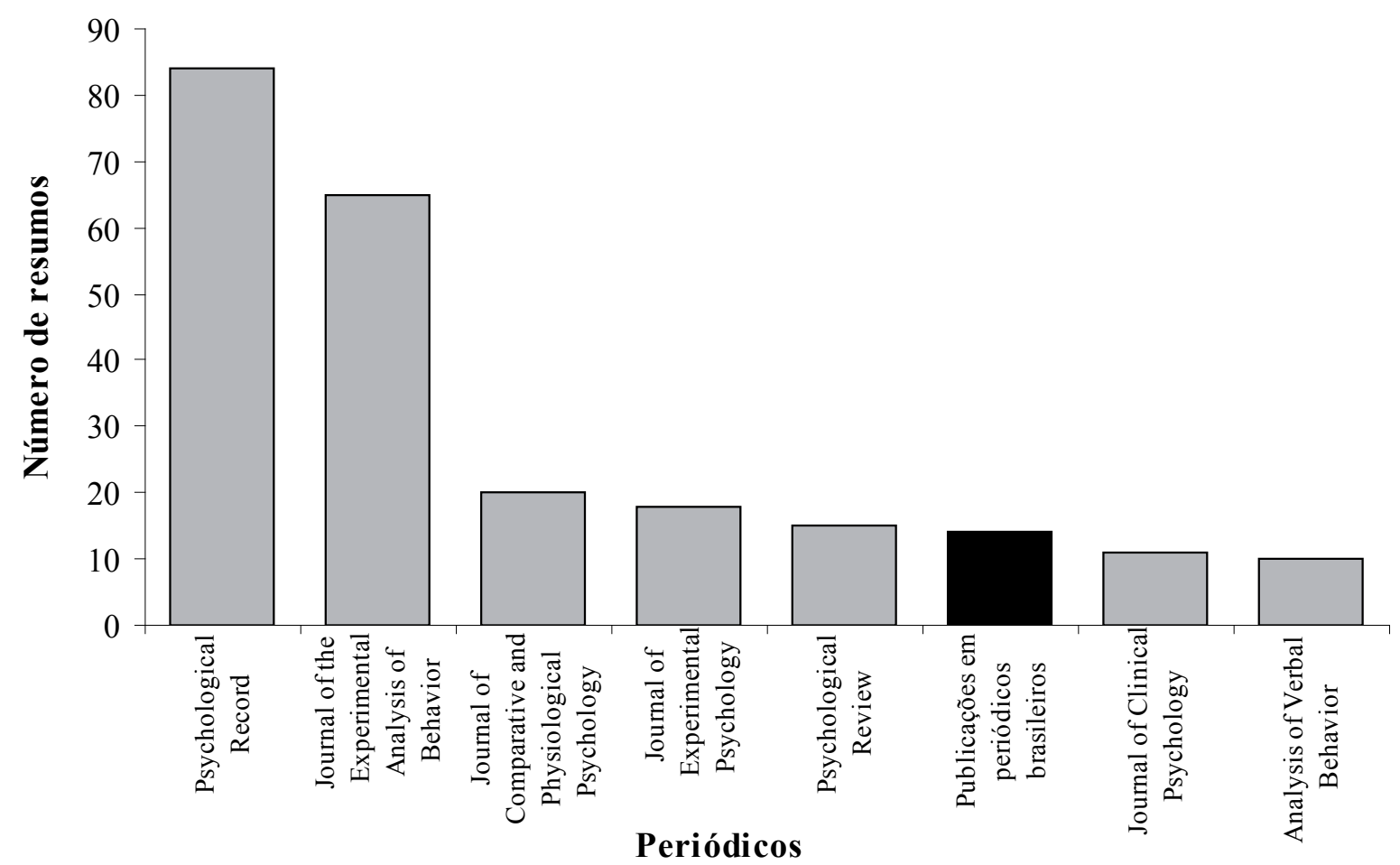

Figura 1. Resultado parcial da busca na base de dados PsycINFO: número de resumos por periódicos.

conhecimento que já foi produzido, de forma a orientar o esclarecimento sobre o assunto, a busca de referências por pesquisadores da área e a organização de novos estudos. De acordo com Morris, Todd, Midgley, Schneider e Johnson (1990), a relevância de pesquisas dessa natureza se baseia principalmente em: evitar a repetição de erros cometidos no passado; ajudar a resolver problemas atuais pelo exame de suas origens e desenvolvimento; ilustrar como uma disciplina vem caminhando e qual é sua tendência para o futuro; descrever como fatores externos, tais como, cultura, política e economia afetaram o crescimento da disciplina, tanto em termos metodológicos quanto teóricos; esclarecer aspectos metodológicos e conceituais da disciplina; e orientar o desenvolvimento de novas pesquisas, e da própria disciplina como um todo.

Apesar das inegáveis contribuições das pesquisas bibliográficas, Choppin (2004) destacou algumas dificuldades relacionadas aos trabalhos que se propõem a realizar a revisão de determinada área do conhecimento. Uma delas refere-se à diversidade de vocabulário e de significados de um mesmo termo, tornando a determinação do objeto de estudo mais imprecisa, o que tem que ser contornado por critérios de seleção bem definidos. Outra reside no acesso restrito e na dificuldade de localização dos materiais quando estes não se encontram em bases de dados disponíveis da WEB.

Os materiais utilizados em pesquisas bibliográficas são as produções de outros autores, sendo que algumas pesquisas recorrem às publicações completas, enquanto outras selecionam apenas os seus resumos. Os resumos são formas bastante concisas de apresentação de pesquisas, os quais, de acordo com normas nacionais e internacionais, devem incluir o tipo de orientação teórica, o objetivo principal de investigação, a metodologia utilizada (procedimento, materiais e sujeitos), os resultados encontrados e as conclusões apresentadas
(Ferreira, 2002). No entanto, nem todos os autores seguem essas instruções, o que junto com as diferentes regras de elaboração de resumos exigidas pelas entidades responsáveis pela divulgação, gera uma heterogeneidade de formas de apresentação, o que dificulta a sua padronização. Devido ao caráter conciso e objetivo dos resumos e dada a ampla variedade de formas de organizá-los, Ferreira alerta para os problemas da realização de pesquisas baseadas somente em resumos, afirmando que não se pode considerá-los como uma representação fidedigna de tudo o que foi estudado na pesquisa. Portanto, o mais indicado, segundo esse autor, seria a leitura do relato completo dos trabalhos quando esses estiverem disponíveis.

A despeito de tais limitações, os trabalhos que utilizam resumos apresentam algumas vantagens. A principal delas é que várias pesquisas não são publicadas em periódicos, estando muitas vezes registradas apenas nos resumos de apresentações em eventos científicos. Nesses casos, a opção por revisar somente as formas completas de apresentação excluiria uma parcela importante e considerável da produção. Além disso, a seleção de trabalhos completos exige maior disponibilidade de tempo e de recursos, visto que grande parte dos trabalhos, especialmente as dissertações e teses mais antigas, não está disponível na WEB. Nas pesquisas que selecionam apenas os resumos, o tempo pode ser otimizado, o que permite abranger um maior número de trabalhos e fornecer um quadro mais completo sobre a área. Quando o objetivo principal de uma dada pesquisa é caracterizar uma determinada área, em um período de tempo mais amplo, e com um maior número de fontes de materiais, incluindo apresentações em congressos, o acesso aos resumos é a opção mais viável. No entanto, se o objetivo, por exemplo, é detalhar metodologias específicas, ou seja, descrever os participantes, os materiais, os locais e os procedimentos de cada pesquisa, os trabalhos completos 
apresentam mais informações e são priorizados. Assim, entende-se que não existe uma melhor opção para todos os casos. A escolha pelo acesso aos resumos ou aos trabalhos completos depende dos objetivos da pesquisa bibliográfica a ser realizada.

Ciente das vantagens e limitações de pesquisas bibliográficas baseadas em resumos, no presente estudo optou-se por acessar apenas os resumos das pesquisas para abranger o maior número possível de trabalhos sobre equivalência de estímulos produzidos no Brasil no período estabelecido. A revisão dos estudos sobre equivalência de estímulos caracteriza-se como um instrumento útil para a atualização dos pesquisadores e familiarização daqueles que podem se beneficiar com os instrumentos, conhecimentos e estratégias de intervenção que vêm sendo produzidos por pesquisadores da Análise do Comportamento. Sendo assim, o objetivo do presente estudo foi identificar, categorizar e analisar os resumos de trabalhos sobre equivalência de estímulos produzidos e publicados no Brasil entre 1997 e 2007 em anais de congressos, artigos de periódicos e dissertações e teses.

\section{Método}

Foram selecionados os resumos que relatam pesquisas teóricas, empíricas ou bibliográficas, que têm em seu corpo pelo menos uma das seguintes palavras: equivalência; classe de estímulo; equivalente; redes relacionais; responder relacional; pares associados ou transitividade e que façam referência à formação e/ou ao teste de relações de equivalência.

Os periódicos nacionais que puderam ser acessados pelas bases de dados CAPES, PEPSIC, INDEXPSI, LILACS e PsycINFO foram selecionados para a realização da busca. Os volumes e números dos periódicos pesquisados foram aqueles que estavam disponíveis ao livre acesso pela internet até a última data de pesquisa (julho de 2008). Por meio do portal de periódico da CAPES, foram localizados os periódicos da área da Psicologia e da Educação. Em seguida, foram realizadas pesquisas individuais, com base nas palavras-chave, em todos os periódicos selecionados. Nas demais bases de dados, foram realizadas outras buscas com as mesmas palavras-chave. Os cadernos, anais e CD-ROM consultados são os dos seguintes eventos nacionais: Encontro Brasileiro de Psicoterapia e Medicina Comportamental (ABPMC) e Reunião Anual da Sociedade Brasileira de Psicologia (SBP). Os resumos de dissertações e teses foram pesquisados por meio da base de dados da CAPES.

Todos os resumos encontrados foram lidos e aqueles que atendiam aos critérios estabelecidos foram selecionados e categorizados de acordo com a universidade, o meio de divulgação, os autores, o ano de publicação, o tipo de pesquisa, os participantes, o objetivo principal e as palavras-chave. Dentre os resultados obtidos, foram excluídos os trabalhos que não apresentavam resumo. Os resultados de algumas pesquisas foram publicados mais de uma vez e, nesses casos, foram mantidas todas as referências sobre a pesquisa, ou seja, uma mesma pesquisa foi contabilizada em todas as vezes que foi publicada.

Para a categorização de participantes foram utilizadas as palavras apresentadas nos resumos. Os objetivos, no entanto, não puderam ser categorizados somente pelas palavras a eles relacionadas porque muitos objetivos não foram claramente identificados e as palavras utilizadas pelos autores diferiram consideravelmente. Dessa forma, foram escolhidas palavras amplas que pudessem englobar várias palavras/expressões com funções semelhantes e assim possibilitar a reunião de resumos. A Tabela 1 apresenta as categorias que envolveram objetivos com palavras diferentes de seus rótulos junto às respectivas palavras. Salienta-se, assim, que a categorização dos objetivos não pôde ser tão precisa quanto a de participantes porque exigiu o agrupamento de palavras diferentes nas categorias e a inclusão de um dado resumo em somente uma das categorias estabelecidas. Optou-se pela definição de apenas um registro por resumo porque, de certa forma, todos os trabalhos sobre o tema são interligados e, em última instância, apresentam objetivos próximos; se todos fossem considerados, não seria possível uma comparação entre eles. Para que cada trabalho tivesse apenas um registro de objetivo, foi necessária a escolha daquele considerado como o principal. Tal escolha e a eliminação dos demais objetivos foram realizadas segundo especificações dos autores ou critérios das pesquisadoras, quais sejam: identificação explícita e coerência com o método e os resultados. Para aumentar a fidedignidade dos dados apresentados, todas as palavraschave citadas pelos autores foram registradas e reunidas possibilitando mais uma forma de análise.

\section{Resultados e Discussão}

Foram selecionados 655 resumos que atendiam aos critérios estabelecidos. Destes, 44 são resumos de trabalhos publicados em periódicos; 136, de dissertações de mestrado e teses de doutorado; e 475, de pesquisas apresentadas em eventos nacionais. A análise dos trabalhos apresentados, no ano de 2005, nos congressos ABPMC e da SBP revelou que, em média, 18\% das pesquisas em Análise Experimental do Comportamento estiveram relacionadas ao estudo da equivalência de estímulos. Ainda nesses eventos, cerca de 2,5\% de tudo que foi publicado em 2005 corresponde a pesquisas sobre esse tema. A Figura 2 apresenta o número de resumos de artigos, dissertações e teses, e de trabalhos apresentados nos congressos da ABPMC e SBP, sobre equivalência de estímulos, publicados a cada ano.

Observa-se, na Figura 2, que, no total, o número de publicações brasileiras sobre equivalência de estímulos, nas fontes pesquisadas, aumentou de 1997 a 2003, apresentou um grande pico em 2004, caiu nos dois anos seguintes e no ano de 2007 teve uma grande recuperação. Esse efeito pode ser observado nos dados dos Congressos da ABPMC (com exceção do expressivo aumento em 2007). O grande número de publicações no congresso da ABPMC em 2004 pode ser justificado pelo fato de que, nesse ano, o evento foi realizado junto com o da Association for Behavior Analysis atraindo a participação de mais pesquisadores. O número de apresentações nos eventos da SBP manteve-se relativamente constante de 1999 a 2003, caiu nos próximos três anos e aumentou em 2007. O número de dissertações e teses publicadas foi maior em 2007, seguido por 2003 e 2004; e menor em 1998 e 1999. Além do aumento do número de pesquisadores interessados 
Tabela 1. Categorias que envolveram objetivos com palavras diferentes de seus rótulos e as respectivas palavras.

Rótulo da categoria $\quad$ Palavras reunidas sob cada rótulo

Ensino de matemática

Classes sequenciais/relações ordinais

Comportamento verbal

Avaliação e/ou ensino de leitura e/ou escrita

Avaliação e/ou ensino de repertório simbólico

Variações de procedimentos

Modificação de classes

Procedimentos alternativos ao matching-tosample

Ensino de outras habilidades
Contagem; conceito de número; manuseio de dinheiro; problemas aritméticos; conceito de proporção; subtração; classes de frações; treino numérico-numérico

Encadeamento de resposta; relações sintáticas e numéricas; sobreposição de pares de estímulos

Teste da mediação verbal; operantes verbais; influências de verbalizações em dupla

Discriminação de unidades mínimas; leitura generalizada recombinativa; ensino combinado de cópia, ditado e oralização; consciência fonológica; leitura em Braille

Reestabelecimento da nomeação; estabelecimento de correspondência simbólica com estímulos auditivos; reabilitação de relações arbitrárias

Estruturas de treino; tamanho das classes; característica dos participantes; exclusão; exposição prévia; sequência de treino e/ou testes; característica dos estímulos; fading; relações de controle; tipo de treino; formas de análise dos dados; topografia da resposta; tipo de relação e de escolha; critério de aprendizagem; intervalo entre tentativas

Reorganização de classes; reversões de discriminações condicionais de linha de base

Go/no-go; discriminações simples simultâneas com estímulos compostos; discriminações simples (reforçador ou resposta específico por classe); pares associados; aprendizagem observacional; pareamento consistente; reversões sucessivas de discriminações simples; sequências intraverbais para cada grupo de estímulos; autodiscriminação; reforçamento automático e direto

Ciências; LIBRAS; medida de tempo; leitura musical; alfabeto digital no tema, relaciona-se o crescimento de dissertações/teses sobre equivalência de estímulos com o início de novos programas de pós-graduação. Os artigos de periódicos não apresentam variações expressivas do número de publicações nos diferentes anos, o que não permite estimar uma tendência. No geral, as reuniões anuais da SBP contaram com o maior número de trabalhos sobre o tema.

Observa-se, ainda na Figura 2, que, com exceção dos últimos três anos, não parece haver uma relação entre o número de dissertações e teses produzidas e de apresentações em eventos. Tal constatação leva à hipótese de que algumas pesquisas geram uma grande produção, com mais de uma publicação em eventos e que, provavelmente, nesses eventos, exista também uma grande participação de trabalhos sem uma relação direta com os programas de mestrado e doutorado. Além disso, verifica-se que o número de publicações em periódicos, com exceção de 2006, é sempre inferior ao número de dissertações ou teses produzidas em um dado ano. Ou seja, muitas pesquisas desenvolvidas nos programas de mestrado e doutorado não são transformadas em artigos científicos ou não estão publicadas em periódicos acessíveis pela WEB nas bases de dados selecionadas para o presente estudo.

Até a última consulta (julho de 2008), estavam disponíveis no Portal de Periódicos da CAPES (Psicologia e Educação) e no PEPSIC os periódicos citados na Tabela 2. Além destes, foram incluídos na busca os periódicos indexados no INDEXPSI, LILACS e PsycINFO, cujos nomes não estão incluídos na tabela porque a maioria dos resumos selecionados foram encontrados nas duas primeiras bases (CAPES e PEPSIC) e porque nas demais houve dificuldade de acesso a tais nomes ou uma grande extensão de periódicos contemplados. A Tabela 2 apresenta o nome e o ISSN dos periódicos, o número de trabalhos selecionados e os anos disponíveis para busca dentro do período de interesse para o presente estudo. Para enquadrar a tabela nos limites de espaço, foram excluídos da lista os periódicos que tinham em seu nome a palavra Psicanálise e o nome de grande parte dos demais periódicos foi abreviado.

Observa-se, na Tabela 2, que um grande número de periódicos da área da Psicologia e da Educação pode ser acessado 


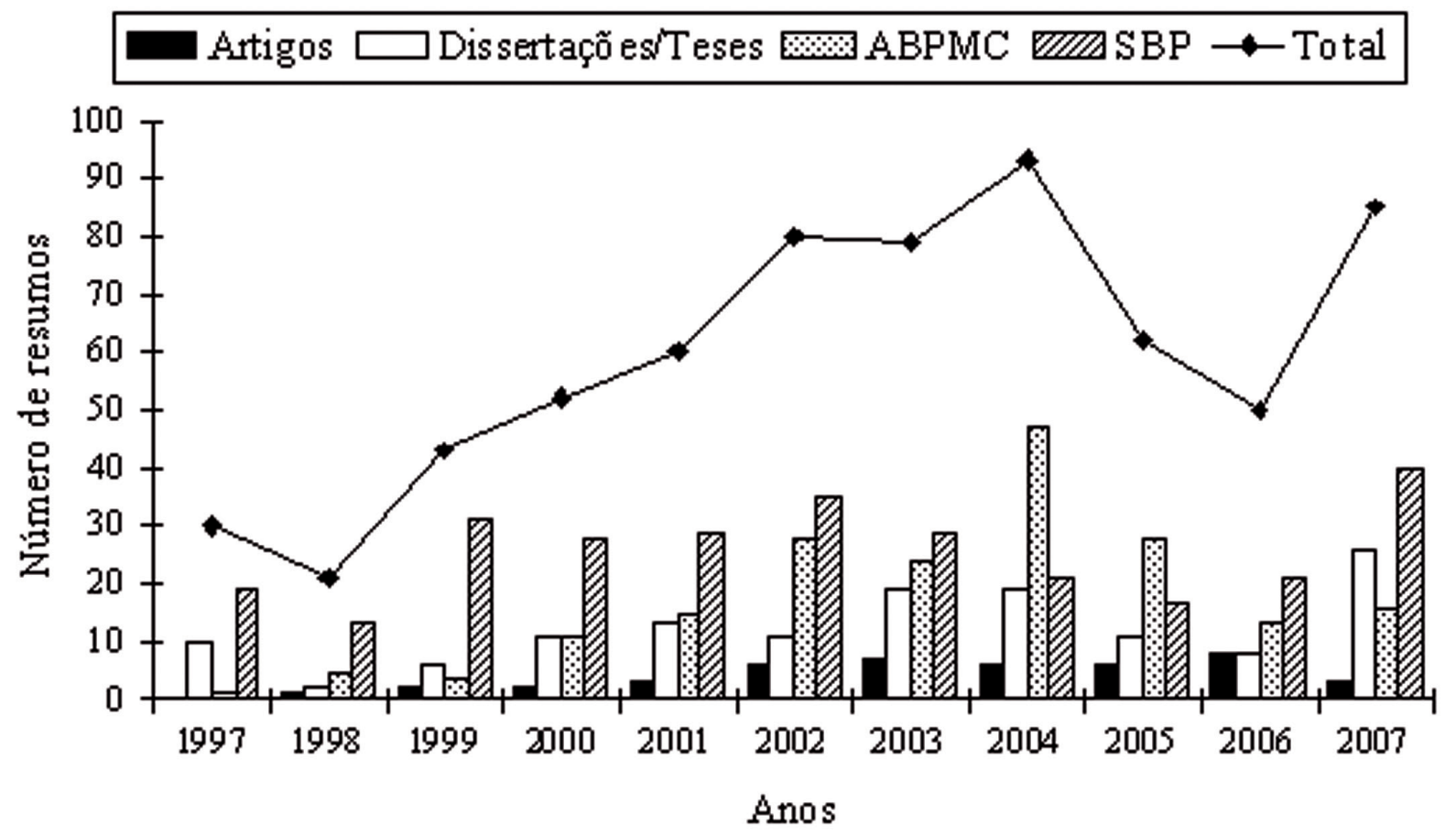

Figura 2. Número de resumos de artigos, dissertações e teses e de trabalhos apresentados nos congressos da ABPMC e SBP, sobre equivalência de estímulos, publicados a cada ano.

na WEB. Entretanto, grande parte deles disponibiliza apenas alguns de seus volumes. Verifica-se, ainda, que dentre os 67 periódicos consultados, apenas 13 publicaram trabalhos sobre equivalência de estímulos, sendo que desses, somente dois são periódicos da área da Educação. O periódico com o maior número de publicações (nove) sobre o tema é Estudos de Psicologia (Natal), seguido por Interação em Psicologia, e Psicologia: Teoria e Pesquisa (ambos com sete publicações cada).

Os resumos selecionados no estudo aqui descrito foram distribuídos em três grupos: relatos de pesquisas com participantes humanos, relatos de pesquisas com animais e trabalhos teóricos, sendo que o número de resumos em cada grupo é de 516, 41 e 98 respectivamente. A Tabela 3 apresenta o número de publicações sobre equivalência de estímulos por universidade e tipo de pesquisa. Nessa tabela, os trabalhos estão agrupados segundo o número de universidades que participaram de sua publicação (uma única universidade, duas ou três).

A partir da Tabela 3, nota-se que o maior número de publicações é de relatos de pesquisas com participantes humanos, seguidos pelos trabalhos teóricos. Existem várias pesquisas com animais envolvidas na investigação dos pré-requisitos para a formação de classes de equivalência (e.g., Goulart, Galvão \& Barros, 2003). Entretanto, deve-se ressaltar que no presente estudo foram selecionadas somente aquelas que apresentavam as palavras definidas como critério. Vários estudos com animais, que investigam esse tema, buscam a verificação da emergência das relações, mas atualmente ainda é difícil a demonstração de transitividade (Goulart, 2005). Dessa forma, esta e outras palavras que se referem à formação de classes de equivalência nem sempre são incluídas nos resumos, o que justifica o baixo número de trabalhos com animais encontrados.
Observa-se ainda, na Tabela 3, que a maioria das instituições em que se conduzem investigações sobre equivalência de estímulos são universidades federais. As universidades estaduais e particulares, por sua vez, quando pesquisam o tema, mesmo que com um número inferior ao das federais, apresentam uma grande produção, como é o caso, por exemplo, da UEL e da PUC-SP, respectivamente. De acordo com os dados da Tabela 3, 37 trabalhos não têm identificação da instituição, o que se deve, em parte, à ausência da menção à afiliação dos autores na maioria dos resumos de artigos de periódicos. A base de dados do PsycINFO destaca-se nesse aspecto porque, no período pesquisado, independente do periódico de que faz parte o resumo apresentado, foi possível a verificação da afiliação dos autores. Apesar disso, foi constatado pelo menos um resumo cuja afiliação provavelmente relacionava-se à instituição em que os autores cursaram a pós-graduação e não àquela em que desenvolveram a pesquisa citada. Esses dados indicam haver necessidade de maior padronização de modelos de resumo para que a busca possa ser facilitada e uniformizada.

De acordo com a Tabela 3, a maioria dos trabalhos (545 dos 655) é realizada com a participação de apenas uma instituição. Considerando somente os 545 trabalhos, a UFPA e a UFSCAR apresentaram os maiores números de publicações, totalizando 117 resumos de cada uma. Ainda quanto aos trabalhos de uma única instituição, os maiores números de publicações com humanos, animais e de trabalhos teóricos são, respectivamente, da UFSCAR, UFPA e UFSCAR. Podese observar, na porção da Tabela 3 referente aos trabalhos realizados em parceria, que houve a participação de pesquisadores estrangeiros em pelo menos 11 trabalhos. No que se refere às publicações com a participação de duas instituições, verifica-se que a UFPA e a UFSCAR apresentam mais publicações totais, com participantes humanos e com animais. 
Tabela 2. Nome e ISSN dos periódicos acessados no Portal da CAPES e no PEPSIC, anos disponíveis para busca e número de trabalhos selecionados $\left(\mathrm{N}^{\circ}\right)$.

\begin{tabular}{|c|c|c|c|c|c|c|c|}
\hline \multirow[b]{2}{*}{ Nome do periódico } & \multicolumn{3}{|c|}{ Dados } & \multirow[b]{2}{*}{ Nome do periódico } & \multicolumn{3}{|c|}{ Dados } \\
\hline & $\begin{array}{l}\text { ISSN do } \\
\text { periódico }\end{array}$ & $\begin{array}{l}\text { Anos dis- } \\
\text { poníveis }\end{array}$ & $\mathbf{N}^{0}$ & & $\begin{array}{l}\text { ISSN do } \\
\text { periódico }\end{array}$ & $\begin{array}{l}\text { Anos dis- } \\
\text { poníveis }\end{array}$ & $\mathbf{N}^{\mathbf{o}}$ \\
\hline Aletheia & $1413-0394$ & 2004-2007 & 0 & Psicologia da Educação & $1414-6975$ & 2004-2005 & 1 \\
\hline Arq. Bras. de Psicologia & $0100-8692$ & 2003-2007 & 3 & Psicologia e Sociedade & $0102-7182$ & $2002-2007$ & 0 \\
\hline $\begin{array}{l}\text { Avaliação: Revista da } \\
\text { Aval. da Educ. Superior }\end{array}$ & $1414-4077$ & 2007 & 0 & Psicologia em Estudo & $1413-7372$ & $2001-2007$ & 1 \\
\hline Avaliação Psicológica & $1677-0471$ & $2002-2007$ & 0 & Psicologia em Revista & $1677-1168$ & $2002-2007$ & 0 \\
\hline Boletim de Psicologia & $0006-5943$ & $2005-2007$ & 0 & Psic. Esc. Educacional & $1413-8557$ & $1997-2007$ & 0 \\
\hline $\begin{array}{l}\text { Cadernos de Psicologia } \\
\text { Social do Trabalho }\end{array}$ & $1516-3717$ & $1998-2007$ & 0 & Psicologia Hospitalar & $1677-7409$ & 2004-2006 & 0 \\
\hline Cad. de Psicopedagogia & $1676-1049$ & 2004-2007 & 0 & Psic. para Amér. Latina & $1870-350 X$ & $2002-2007$ & 0 \\
\hline Ciência e Educação & $1516-7313$ & $1998-2007$ & 0 & Psicologia USP & 0103-6564 & $1997-2005$ & 0 \\
\hline Construção Psicopedag. & $1415-6954$ & $2005-2007$ & 0 & Psicólogo Informação & $1415-8809$ & 2004 & 1 \\
\hline Educação & 0101-9031 & $2000-2007$ & 0 & Psic.: Teoria e Pesquisa & $0102-3772$ & $2000-2007$ & 7 \\
\hline Educação Especial & $1008-270 X$ & $2000-2005$ & 0 & Psic.: Teoria e Prática & $1516-3687$ & $1999-2007$ & 1 \\
\hline Educação e Pesquisa & $1517-9702$ & $1999-2007$ & 0 & Psicologia Argumento & $0103-7013$ & $2005-2007$ & 0 \\
\hline Educação e Sociedade & $0101-7330$ & $1997-2007$ & 0 & PsicoUSF & $1413-8271$ & $2001-2007$ & 0 \\
\hline $\begin{array}{l}\text { Educação Temática Digi- } \\
\text { tal (ETD) }\end{array}$ & $1676-2592$ & $1999-2007$ & 0 & $\begin{array}{l}\text { Rev. Bras. de Crescim. e } \\
\text { Desenvolv. Humano }\end{array}$ & 0104-1282 & 2007 & 0 \\
\hline
\end{tabular}

Nota. Os anos foram classificados como disponíveis quando apresentavam pelo menos um número do periódico, assim, a presença de um dado ano na tabela não significa que todos os seus números foram acessados. 
Tabela 2 (continuação). Nome e ISSN dos periódicos acessados no Portal da CAPES e no PEPSIC, anos disponíveis para busca e número de trabalhos selecionados $\left(\mathrm{N}^{\circ}\right)$.

\begin{tabular}{|c|c|c|c|c|c|c|c|}
\hline \multirow[b]{2}{*}{ Nome do periódico } & \multicolumn{3}{|c|}{ Dados } & \multirow[b]{2}{*}{ Nome do periódico } & \multicolumn{3}{|c|}{ Dados } \\
\hline & $\begin{array}{l}\text { ISSN do } \\
\text { periódico }\end{array}$ & $\begin{array}{l}\text { Anos dis- } \\
\text { poníveis }\end{array}$ & $\mathbf{N}^{\mathbf{o}}$ & & $\begin{array}{l}\text { ISSN do } \\
\text { periódico }\end{array}$ & $\begin{array}{l}\text { Anos dis- } \\
\text { poníveis }\end{array}$ & $\mathbf{N}^{\mathbf{o}}$ \\
\hline $\begin{array}{l}\text { Ensaio: Aval. e Políticas } \\
\text { Públicas em Educação }\end{array}$ & 0104-4036 & $2004-2007$ & 0 & $\begin{array}{c}\text { Revista brasileira de } \\
\text { educação }\end{array}$ & $1413-2478$ & $1997-2007$ & 0 \\
\hline Escritos sobre Educação & $1677-9843$ & $2005-2006$ & 0 & Rev. Bras. Educ. Espec. & $1413-6538$ & $2005-2007$ & 1 \\
\hline Estilos da Clínica & $1415-7128$ & $2003-2007$ & 0 & Rev. Bras. Educ. Méd. & $0100-5202$ & $2002-2007$ & 0 \\
\hline $\begin{array}{l}\text { Estudos de Psicologia } \\
\text { (Campinas) }\end{array}$ & 0103-166X & 2004-2007 & 0 & $\begin{array}{l}\text { Revista Brasileira de } \\
\text { Orientação Profissional }\end{array}$ & $1679-3390$ & $2003-2007$ & 0 \\
\hline $\begin{array}{l}\text { Estudos de Psicologia } \\
\text { (Natal) }\end{array}$ & 1413-294X & $1997-2007$ & 9 & $\begin{array}{l}\text { Rev. Bras. de Terapia } \\
\text { Comport. e Cognitiva }\end{array}$ & $1517-5545$ & $1999-2007$ & 5 \\
\hline $\begin{array}{l}\text { Estudos e Pesquisas em } \\
\text { Psicologia }\end{array}$ & $1808-4281$ & $2004-2007$ & 0 & $\begin{array}{l}\text { Revista Brasileira de } \\
\text { Terapias Cognitivas }\end{array}$ & $1808-5687$ & $2005-2007$ & 0 \\
\hline Gestão em Ação & $1808-124 \mathrm{X}$ & $2000-2006$ & 0 & Rev. da Fac. Educação & $0102-2555$ & $1997-1998$ & 0 \\
\hline Imaginário & $1413-666 X$ & $2005-2007$ & 0 & Revista da SBPH & $1516-0858$ & 2004-2007 & 0 \\
\hline Interação em Psicologia & $1981-8076$ & $1997-2007$ & 7 & Revista da SPAGESP & $1677-2970$ & $2003-2007$ & 0 \\
\hline $\begin{array}{l}\text { Interações: Estudo e Pes- } \\
\text { quisa em Psicologia }\end{array}$ & $1413-2907$ & $1997-2006$ & 0 & Revista de Etologia & $1517-2805$ & $2005-2006$ & 0 \\
\hline $\begin{array}{l}\text { Interface: Comunicação, } \\
\text { Saúde, Educação }\end{array}$ & $1807-5762$ & $1997-2007$ & 0 & $\begin{array}{l}\text { Rev. do Departamento de } \\
\text { Psicologia UFF }\end{array}$ & $0104-8023$ & $2005-2007$ & 0 \\
\hline $\begin{array}{l}\text { Latin-Amer. Journal of } \\
\text { Fund. Psychopathology }\end{array}$ & $1677-0358$ & 2007 & 0 & $\begin{array}{l}\text { Rev. Eletr. do Mestrado } \\
\text { em Educ. Ambiental }\end{array}$ & $1517-1256$ & $2004-2007$ & 0 \\
\hline $\begin{array}{l}\text { Memorandum: Memória } \\
\text { Hist. em Psic. }\end{array}$ & $1676-1669$ & $2001-2007$ & 0 & $\begin{array}{c}\text { Revista HISTEDBR } \\
\text { Online }\end{array}$ & $1676-2584$ & $2000-2007$ & 0 \\
\hline Mental & $1679-4427$ & $2003-2007$ & 0 & Rev. Interameric. Psic. & $0034-9690$ & $2006-2007$ & 0 \\
\hline Perspectiva & $0102-5473$ & $1997-2007$ & 0 & Rev. Paul. Educ. Física & $0102-7549$ & $1997-2004$ & 0 \\
\hline $\begin{array}{l}\text { Psicologia: Ciência e } \\
\text { Profissão }\end{array}$ & $1414-9893$ & $2000-2007$ & 1 & $\begin{array}{l}\text { Revista Elet. Saúde Men- } \\
\text { tal Álcool e Drogas }\end{array}$ & $1806-6976$ & $2005-2007$ & 0 \\
\hline Psic: Pesq. e Trânsito & $1808-9100$ & $2005-2006$ & 0 & Temas em Psic. da SBP & $1413-389 X$ & 2004-2005 & 4 \\
\hline Psic.: Reflexão e Crítica & 0102-7972 & $1997-2007$ & 3 & Trab., Educ. e Saúde & $1981-7746$ & $2003-2005$ & 0 \\
\hline Psic: Rev. Vetor Editora & $1676-7314$ & $2002-2007$ & 0 & Vínculo: Rev. NESME & $1806-2490$ & $2004-2007$ & 0 \\
\hline Psicologia Clínica & 0103-5665 & $2000-2007$ & 0 & & & & \\
\hline
\end{tabular}

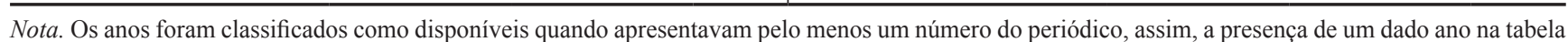
não significa que todos os seus números foram acessados. 
Apesar do grande número de resumos encontrados, a maioria das pesquisas sobre equivalência de estímulos tem sido realizada em poucos estados brasileiros, sendo os principais representantes (quanto ao número de produções dentro dos critérios da presente pesquisa), os estados de São Paulo, Pará, Goiás, Paraná e Santa Catarina. No geral, verifica-se que vários trabalhos realizados com a participação de mais de uma instituição limitam-se a universidades de um mesmo estado, como é o caso de alguns trabalhos de Goiás e de São Paulo. Entretanto, a distância geográfica não é impeditivo para a união de instituições em uma mesma pesquisa, o que é demonstrado pelos frequentes trabalhos realizados em conjunto pela UFPA e UFSCAR.

A Tabela 4 apresenta três conjuntos de categorias de pesquisas realizadas com participantes humanos e a porcentagem de pesquisas realizadas em cada uma delas. Na Tabela 4, o primeiro conjunto de categorias relaciona-se à faixa etária dos participantes; o segundo, às características principais dos participantes; e o terceiro, aos objetivos principais. A somatória dos dados de cada conjunto, isoladamente, refere-se à

Tabela 3. Número de publicações, de cada universidade, de pesquisas com participantes humanos (H), não humanos (NH) e revisões teóricas (T)

\begin{tabular}{|c|c|c|c|c|c|c|c|}
\hline \multicolumn{8}{|c|}{ Número de trabalhos publicados } \\
\hline Universidades & $\mathbf{H}$ & NH & $\mathbf{T}$ & Universidades & $\mathbf{H}$ & NH & $\mathbf{T}$ \\
\hline 1 Univ & & & & 2 Univ & & & \\
\hline PUC-SP & 23 & 0 & 6 & UCG / UFG & 7 & 0 & 0 \\
\hline $\mathrm{UCG}$ & 17 & 0 & 1 & $\mathrm{UCG} / \mathrm{UnB}$ & 1 & 0 & 1 \\
\hline UEL & 54 & 0 & 6 & UEL / UFSCAR & 3 & 0 & 0 \\
\hline UFES & 1 & 0 & 1 & UEL / USP & 0 & 0 & 2 \\
\hline UFMG & 2 & 0 & 1 & UFMG / UnB & 3 & 0 & 1 \\
\hline UFPA & 76 & 25 & 16 & UFPA / UFSCAR & 14 & 2 & 1 \\
\hline UFSC & 23 & 0 & 0 & UFSCAR / UNESP & 10 & 0 & 0 \\
\hline UFSCAR & 91 & 4 & 22 & UFSCAR / UNICENP & 3 & 0 & 0 \\
\hline UNAMA & 2 & 0 & 1 & UFSCAR / USP & 7 & 0 & 0 \\
\hline UnB & 39 & 1 & 4 & UNESP / USP & 2 & 0 & 1 \\
\hline UNESP & 34 & 0 & 1 & UPM / USP & 7 & 0 & 2 \\
\hline UNIT & 0 & 0 & 2 & Outras & 15 & 0 & 6 \\
\hline UNIVALI & 4 & 0 & 0 & 3 Univ & & & \\
\hline UPM & 8 & 0 & 1 & UCG / UnB / UFG & 1 & 0 & 0 \\
\hline USP & 28 & 4 & 4 & UEL / USP / UFSCAR & 1 & 0 & 0 \\
\hline Não Identificada & 21 & 3 & 13 & UFMG / UnB / UFPA & 1 & 0 & 0 \\
\hline Outras & 3 & 0 & 3 & UFPA / UFSCAR / UNAMA & 1 & 0 & 0 \\
\hline 2 Univ & & & & UFSCAR / UFPA / Estrang. & 1 & 0 & 0 \\
\hline Estrang. / UFPA & 4 & 0 & 1 & UFSCAR / UnB / UFPA & 0 & 0 & 1 \\
\hline Estrang. / UFSCAR & 1 & 2 & 0 & UFSCAR / UNESP / USP & 5 & 0 & 0 \\
\hline Estrang. / USP & 2 & 0 & 0 & USP / UNICENP / UFSCAR & 1 & 0 & 0 \\
\hline
\end{tabular}

Nota. 1 Univ: trabalhos com autores de uma única universidade; 2 Univ: trabalhos com a participação de duas universidades; 3 Univ: trabalhos com a participação de três universidades; Outras: universidades ou conjunto de universidades com menos do que duas publicações. Estrang.: Estrangeiro; PUCSP: Pontifícia Universidade Católica de São Paulo; UCG: Universidade Católica de Goiás; UEL: Universidade Estadual de Londrina; UFES: Universidade Federal do Espírito Santo; UFG: Universidade Federal de Goiás; UFMG: Universidade Federal de Minas Gerais; UFPA: Universidade Federal do Pará; UFSC: Universidade Federal de Santa Catarina; UFSCAR: Universidade Federal de São Carlos; UNAMA: Universidade da Amazônia; UnB: Universidade de Brasília; UNESP: Universidade Estadual Paulista; UNICENP: Universidade Positivo; UNIT: Centro Universitário do triângulo-MG; UNIVALI: Universidade do Vale do Itajaí; UPM: Universidade Presbiteriana Mackenzie; USP: Universidade de São Paulo. 
totalidade dos trabalhos encontrados; ou seja, cada resumo foi classificado de acordo com cada conjunto de categorias e com apenas um registro em cada um deles.

Observa-se, na Tabela 4, que a maior parte das pesquisas com humanos foi realizada, quanto à faixa etária, com crianças ou adolescentes; em relação às características dos participantes, com universitários; e quanto ao objetivo principal, com a avaliação ou ensino de leitura e escrita. Com menos resumos, mas com porcentagens superiores a $6 \%$, podem também ser citados os trabalhos: com jovens ou adultos (faixa etária); com pessoas com dificuldades de aprendizagem, pré-escolares, estudantes regulares e com desenvolvimento atípico (características dos participantes); sobre variações de procedimento, procedimentos alternativos ao MTS, transferência de função, classes sequenciais e ensino de matemática (objetivo principal). Os participantes menos estudados, quanto à faixa etária, são os idosos e os bebês e, quanto às características, bilíngues, pessoas com diferenças de peso, contaminados por chumbo e deficientes visuais. Foram observadas pequenas porcentagens de resumos que tratavam principalmente do ensino de pré-requisitos para a formação de classes, do controle contextual, do comportamento verbal e do ensino de mais de uma habilidade acadêmica.

Pode-se afirmar, portanto, no que se refere aos critérios do presente estudo, que as pesquisas com humanos sobre equivalência de estímulos têm focalizado, com maior frequência, o desenvolvimento de estratégias de ensino e a investigação de variáveis que interferem na formação de classes de equivalência. Ao comparar os dados da Tabela 4 com os da Tabela 2, verifica-se que apesar da expressiva produção e grande aplicabilidade do paradigma da equivalência de estímulos à Educação, muito pouco tem sido publicado em periódicos desta área. Ou seja, a produção é grande, mas provavelmente a população que mais se beneficiaria dela não tenha acesso a esse conhecimento e tecnologia.

As categorias de participantes e de objetivos foram relacionadas compondo a Tabela 5. Nessa tabela, cada categoria com mais do que 5\% de resumos, segundo a Tabela 4, foi relacionada com as demais que atendiam a esse mesmo critério. Nas linhas foram dispostas as categorias de participantes (formadas pela faixa etária seguida pelas características) e, nas colunas, as categorias de objetivos. Verifica-se, na Tabela 5, que os trabalhos sobre leitura e escrita envolveram uma grande variedade de participantes, sendo os mais frequentes crianças ou adolescentes com dificuldade de aprendizagem, pré-escolares e estudantes regulares. Os trabalhos sobre variações de procedimentos e procedimentos alternativos ao MTS, por sua vez, contaram principalmente com jovens ou adultos universitários. Pesquisas cujo objetivo foi a investigação da transferência de controle envolveram, com maior frequência, crianças ou adolescentes pré-escolares e jovens ou adultos universitários.

Pode-se observar, ainda na Tabela 5, que crianças ou adolescentes foram os participantes mais frequentes das pesquisas sobre ensino de matemática e sobre classes sequenciais. Constata-se que, em pesquisas com objetivos de ensino de habilidades acadêmicas, os participantes são
Tabela 4. Porcentagem de resumos selecionados em cada categoria de participantes e de objetivos.

\begin{tabular}{|c|c|}
\hline Categoria & $\begin{array}{l}\% \text { de } \\
\text { resumos }\end{array}$ \\
\hline \multicolumn{2}{|l|}{ Faixa Etária dos Participantes } \\
\hline Bebês & 0,8 \\
\hline Crianças ou adolescentes & 50,6 \\
\hline Jovens ou adultos & 25,2 \\
\hline Crianças ou adolescentes e adultos & 5,8 \\
\hline Adultos e idosos & 0,6 \\
\hline Díades & 4,0 \\
\hline Não especificado & 13,0 \\
\hline \multicolumn{2}{|l|}{ Características dos Participantes } \\
\hline Com deficiências múltiplas & 1,4 \\
\hline Com desenvolvimento atípico & 8,7 \\
\hline Com desenvolvimento típico e atípico & 2,7 \\
\hline Com problemas neurológicos & 1,9 \\
\hline Com deficiência auditiva & 4,3 \\
\hline Com deficiência visual & 0,4 \\
\hline Contaminados por chumbo & 0,4 \\
\hline Com distúrbio emocional & 1,2 \\
\hline Com diferenças de peso & 0,4 \\
\hline Com dificuldade de aprendizagem & 17,8 \\
\hline Pré-escolares & 14,3 \\
\hline Estudantes regulares & 12,4 \\
\hline Universitários & 20,0 \\
\hline Bilíngues & 0,2 \\
\hline Não especificado & 13,9 \\
\hline \multicolumn{2}{|l|}{ Objetivos Principais } \\
\hline Ensino de matemática & 7,0 \\
\hline Classes sequenciais / relações ordinais & 7,6 \\
\hline Comportamento verbal & 1,9 \\
\hline Avaliação e/ou ensino de leitura e/ou escrita & 28,3 \\
\hline $\begin{array}{l}\text { Avaliação e/ou estabelecimento de repertório } \\
\text { simbólico }\end{array}$ & 4,5 \\
\hline Equivalência de posição & 2,1 \\
\hline Variações de procedimentos & 18,4 \\
\hline Estabilidade / manutenção de classes & 2,5 \\
\hline Modificação de classes & 2,7 \\
\hline Ensino de pré-requisitos para a formação de classes & 0,8 \\
\hline $\begin{array}{l}\text { Procedimentos alternativos ao matching-to- } \\
\text { sample (MTS) }\end{array}$ & 13,9 \\
\hline Transferência de função ou de controle & 7,8 \\
\hline Controle contextual & 0,8 \\
\hline $\begin{array}{l}\text { Ensino de geografia, ciências ou mais de uma } \\
\text { habilidade acadêmica }\end{array}$ & 1,7 \\
\hline
\end{tabular}


Tabela 5. Porcentagem de resumos em algumas combinações de categorias de participantes e de objetivos.

\begin{tabular}{|c|c|c|c|c|c|c|}
\hline \multirow{2}{*}{ Faixa etária e característica dos participantes } & \multicolumn{6}{|c|}{ Objetivos principais } \\
\hline & Ens Mat & Cla Seq & Lei Esc & Var Pro & Pro Alt & Tra Fun \\
\hline \multicolumn{7}{|l|}{ Crianças ou Adolescentes } \\
\hline Com desenvolvimento atípico & 0 & 0,2 & 1,0 & 0,2 & 1,0 & 0,2 \\
\hline Com dificuldade de aprendizagem & 1,6 & 1,2 & 9,1 & 2,1 & 0,2 & 0,2 \\
\hline Pré-escolares & 1,9 & 0,6 & 5,8 & 1,4 & 1,2 & 1,9 \\
\hline Estudantes regulares & 1,2 & 1,4 & 3,3 & 2,1 & 0 & 0,4 \\
\hline Não especificado & 0 & 0,8 & 0,6 & 1,0 & 0,4 & 0,4 \\
\hline \multicolumn{7}{|l|}{ Crianças ou Adolescentes e Adultos } \\
\hline Com desenvolvimento atípico & 1,4 & 0 & 0 & 0 & 0 & 0 \\
\hline Com dificuldade de aprendizagem & 0,2 & 0 & 0,4 & 0 & 0 & 0 \\
\hline Estudantes regulares & 0 & 0 & 0 & 0,4 & 0 & 0 \\
\hline Não especificado & 0 & 0 & 0 & 0,2 & 0 & 0 \\
\hline \multicolumn{7}{|l|}{ Jovens ou Adultos } \\
\hline Com desenvolvimento atípico & 0,2 & 0 & 0 & 0 & 0 & 0 \\
\hline Com dificuldade de aprendizagem & 0 & 0 & 0,6 & 0 & 0 & 0 \\
\hline Universitários & 0 & 0,4 & 0,4 & 5,0 & 5,6 & 2,1 \\
\hline Não especificado & 0 & 0 & 0 & 1,4 & 1,4 & 0,2 \\
\hline \multicolumn{7}{|l|}{ Não especificado } \\
\hline Com desenvolvimento atípico & 0,2 & 1,4 & 1,0 & 0,2 & 0,2 & 0 \\
\hline Com dificuldade de aprendizagem & 0 & 0,2 & 0,4 & 0 & 0 & 0 \\
\hline Estudantes regulares & 0 & 0 & 0 & 0,6 & 0,8 & 0,2 \\
\hline Não especificado & 0 & 0 & 0,6 & 0,6 & 1,7 & 1,6 \\
\hline
\end{tabular}

Nota. Ens Mat: Ensino de Matemática; Cla Seq: Classes sequenciais / relações ordinais; Lei Esc: Avaliação e/ou ensino de leitura e/ou escrita; Var Pro: Variações de procedimentos; Pro Alt: Procedimentos alternativos ao matching-to-sample (MTS); Tra Fun: Transferência de função ou de controle.

justamente aqueles que se beneficiariam com a tecnologia produzida; ou seja, tais pesquisas realizam-se em contextos mais aplicados. Grande parte das demais pesquisas, por outro lado, priorizam a participação de universitários. É possível também verificar que as porcentagens de participantes não identificados é relativamente alta, especialmente quando se trata de pessoas com desenvolvimento atípico.

Todas as palavras-chave apresentadas pelos autores nos resumos foram também registradas, possibilitando a formulação da Tabela 6. Essa tabela apresenta as palavras-chave mais frequentemente citadas nos resumos de pesquisas com participantes humanos e os respectivos números de citações. Verifica-se, nessa tabela, que as palavras referentes ao contexto educacional correspondem a grande parte das palavras-chave mais frequentes, confirmando, mais uma vez, a dedicação de estudos sobre equivalência de estímulos a essa área. Além disso, observa-se o considerável número de referências ao paradigma da equivalência de estímulos respaldando, ainda que parcialmente, os critérios de seleção de resumos estabelecidos para o presente estudo. Pode-se verificar, também, que alguns autores enfatizam especificidades dos procedimentos utilizados e/ou do objetivo da pesquisa. Por fim, a utilização dessas e outras expressões frequentes entre as palavras-chave indica que diversos autores buscaram explicitar o paradigma envolvido no estudo, características de seus participantes, contextos de aplicação da tecnologia proposta, assim como aspectos das metodologias empregadas.

\section{Considerações Finais}

Nota-se uma grande produção de analistas do comportamento sobre equivalência de estímulos. O número de trabalhos sobre o tema que puderam ser encontrados nos anais de eventos nacionais, nos periódicos de Psicologia e no banco de dados das dissertações de mestrado e teses de doutorado da CAPES é consideravelmente grande. No geral, o acesso aos artigos é um pouco dificultado, em vista da dispersão dos mesmos e da disponibilidade de acesso a somente alguns números na WEB. Observa-se, ainda, que grande parte das pesquisas publicadas conta com a participação dos mesmos autores e das mesmas instituições de pesquisa, ou seja, está concentrada em alguns grupos, o que condiz com a proposta de desenvolvimento de linhas de pesquisa. Nota-se, também, a existência de intercâmbio entre diferentes pesquisadores e instituições, o que é muito positivo para o desenvolvimento da área e atualização do que tem sido pesquisado. 
Tabela 6. Palavras-chave mais citadas nos resumos de pesquisas com participantes humanos e número de citações.

\begin{tabular}{|c|c|c|c|}
\hline Palavras-chave & $\mathbf{n}^{\circ}$ & Palavras-chave & $\mathbf{n}^{\circ}$ \\
\hline Equivalência de estímulos & 238 & Classes de estímulos & 14 \\
\hline Discriminação condicional & 81 & Generalização & 14 \\
\hline Leitura & 58 & Habilidades matemáticas & 14 \\
\hline Equivalência & 51 & Leitura recombinativa & 14 \\
\hline Dificuldades de aprendizagem & 34 & Sala de aula & 14 \\
\hline Classes sequenciais / relações ordinais & 31 & Conceito de número & 13 \\
\hline Escrita & 28 & Pareamento consistente & 13 \\
\hline Ensino por computador & 27 & Unidades menores & 13 \\
\hline Pré-escolares & 24 & CRMTS & 12 \\
\hline Transferência de funções & 23 & Implante coclear & 12 \\
\hline Deficiência auditiva & 20 & Procedimento de exclusão & 12 \\
\hline Escolha de acordo com o modelo & 19 & Controle instrucional & 11 \\
\hline Crianças & 18 & Estímulos auditivos & 11 \\
\hline Comportamento precorrente auxiliar & 17 & Matching-to-sample & 11 \\
\hline Manutenção & 17 & Necessidades educacionais especiais & 11 \\
\hline Relações condicionais & 17 & Tamanho das classes & 11 \\
\hline Relações de equivalência & 17 & Aprendizagem observacional & 10 \\
\hline Classes de estímulos equivalentes & 16 & Discriminação instruída & 10 \\
\hline Deficiência mental & 16 & Funções simbólicas & 10 \\
\hline Estrutura de treino & 15 & Relações numéricas & 10 \\
\hline Nomeação & 15 & Reorganização de classes & 10 \\
\hline
\end{tabular}

Muitos estudos aplicados à Educação têm sido desenvolvidos, apesar de quase nada ser divulgado em eventos ou periódicos dessa área. A investigação de variáveis que afetam o processo de leitura e escrita, e o desenvolvimento de estratégias de intervenção, na maioria das vezes, restringem-se a publicações em eventos e periódicos da Psicologia. Mesmo nas publicações de eventos científicos de Psicologia, a maioria dos trabalhos (incluindo os que propõem estratégias para a Educação) é publicada sob a caracterização de pesquisa em Análise Experimental do Comportamento e pouca referência é encontrada na área destinada à Psicologia Escolar.

Considerando todas as restrições para a realização de um trabalho de revisão da bibliografia, as limitações da metodologia escolhida para a pesquisa aqui descrita e a disponibilidade de prazos e recursos, salienta-se que, embora envolva um grande número de publicações, este é somente um estudo exploratório, a ser atualizado e complementado. Sabe-se, ainda, que os critérios aqui eleitos para seleção de publicações e classificação do material encontrado são passíveis de erros e contestações. No entanto, apesar de todas as limitações e possíveis falhas, considera-se que esta pesquisa pode auxiliar com a apresentação de um panorama geral de quanto, sobre o que e onde têm sido realizadas pesquisas, na última década, sobre equivalência de estímulos. Como um alerta aos pesquisadores, observa-se que nem todos os resumos são claros, completos e objetivos, e que alguns deixam inclusive de citar a instituição de origem dos autores ou dificultam a compreensão do objetivo principal do trabalho.

\section{Referências}

Assis, G. J. A. de, \& Galvão, O. F. (1996). Relações condicionais entre palavras conhecidas. Acta Comportamentalia, 4, 5-22.

Bagaiolo, L. F., \& Micheletto, N. (2004). Fading e exclusão: aquisição de discriminações condicionais e formação de classes de estímulos equivalentes. Temas em Psicologia, 12, 168-185.

Batitucci, J. S. L. (2007). Paradigma de equivalência de estímulos no ensino de leitura de sequências de notas musicais. Dissertação de Mestrado, Universidade de Brasília, Brasília.

Bortoloti, R., \& de Rose, J. C. (2007). Medida do grau de relacionamento entre estímulos equivalentes. Psicologia: Reflexão e Crítica, 20, 252-258.

Carmo, J. S., Silva, L. C. C., \& Figueiredo, R. M. E. (1999). (Orgs.). Dificuldades de aprendizagem no ensino de leitura, escrita e conceitos matemáticos. Belém: UNAMA.

Catania, A. C. (1999). Aprendizagem: comportamento, linguagem e cognição (4a. ed.) (D. G. de Souza, Trad.). Artmed: Porto Alegre. (Trabalho original publicado em 1998).

Choppin, A. (2004). História dos livros e das edições didáticas: sobre o estado da arte. Educação e Pesquisa, 30, 549-566.

D’Oliveira, M. M. H., \& Matos, M. A. (1993). Controle discriminativo na aquisição da leitura: efeito da repetição e variação na posição das sílabas e letras. Temas em Psicologia, 2, 99-108.

de Rose, J. C., de Souza, D. G., \& Hanna, E. S. (1996). Teaching reading and spelling: Exclusion and stimulus equivalence. Journal of Applied Behavior Analysis, 29, 451-469. 
Ferreira, N. S. de A. (2002). As pesquisas denominadas "Estado da Arte". Educação e Sociedade, 23, 257-272.

Figueiredo, R. M. E., Silva, L. C. C., Soares, U. R., \& Barros, R. S. (2001). (Orgs.). Ensino da leitura, escrita e conceitos matemáticos; exercícios de análise do comportamento. Belém: FIDESA/UNAMA.

Goulart, P. R. K. (2005). Considerações acerca da viabilidade de modelos animais para o estudo do comportamento simbólico. Em L. C. Albuquerque (Org.), Estudos do comportamento (pp. 29-43). Belém: EDUFPA.

Goulart, P. R. K., Galvão, O. F., \& Barros, R. S. (2003). Busca de formação de classes de estímulos via procedimento de reversões repetidas de discriminações simples combinadas em macaco-prego (Cebus apella). Interação em Psicologia, 7, 109-119.

Matos, M. A. (1999). Controle de estímulo condicional, formação de classes conceituais e comportamentos cognitivos. Revista Brasileira de Terapia Comportamental e Cognitiva, 1, 159-178.

Medeiros, J. G., \& Nogueira, M. F. (2005). A nomeação de figuras como facilitadora do ler e escrever em crianças com dificuldade de aprendizagem. Psicologia: Teoria e Prática, 7, 107-126.

Morris, E. K., Todd, J. T., Midgley, B. D., Schneider, S. M., \& Johnson, L. M. (1990). The history of behavior analysis: Some historiography and a bibliography. The Behavior Analyst, 13, 131-158

Rossit, R. A. S., \& Ferreira, P. R. S. (2003). Equivalência de estímulos e o ensino de pré-requisitos monetários para pessoas com deficiência mental. Temas em Psicologia, 11, 97-106.

Sidman, M. (1986). Functional analysis of emergent verbal classes. Em T. Thompson \& M. D. Zeiler (Orgs.), Analysis and integration of behavioral units (pp. 213-245). Hillsdale, NJ: Lawrence Erlbaum Associates.
Sidman, M. (2000). Equivalence relations and the reinforcement contingency. Journal of the Experimental Analysis of Behavior, 74, 127-146.

Sidman, M., Kirk, B., \& Willson-Morris, M. (1985). Sixmember stimulus classes generated by conditional-discrimination procedures. Journal of the Experimental Analysis of Behavior, 43, 21-42.

Sidman, M., \& Tailby, W. (1982). Conditional discrimination vs. matching-to-sample: An expansion of the testing paradigm. Journal of the Experimental Analysis of Behavior, 37, 5-22.

Skinner, B.F. (1953). Science and human behavior. New York: Macmillan.

Souza, S. R. de, Goyos, C., Silvares, E. F. M., \& Saunders, R. (2007). Emergence of printing and spelling skills from constructedresponse matching-to-sample instruction (CRMTS). European Journal of Behavior Analysis, 8, 49-64.

Stromer, R., Mackay, H. A., \& Stoddart, L. T. (1992). Classroom applications of stimulus equivalence technology. Journal of Behavioral Education, 2, 225-256.

Wilkinson, K. M., \& McIlvane, W. J. (2001). Methods for studying symbolic behavior and category formation: Contributions of stimulus equivalence research. Developmental Review, 21, 355-374.

Zentall, T. R., \& Smeets, P. M. (Orgs.). (1996). Stimulus class formation in humans and animals. Amsterdam: Elsevier. 